https://idp.uoc.edu

ARTÍCULO

\title{
Estudio de los factores de riesgo en menores infractores para el diseño de intervención social
}

\author{
Amaia Yurrebaso Macho \\ Universidad de Salamanca \\ Eva M. ${ }^{\text {a Picado Valverde }}$ \\ Universidad de Salamanca \\ Begoña Orgaz Baz \\ Universidad de Salamanca
}

\section{Resumen}

Fecha de presentación: mayo de 2021

Fecha de aceptación: noviembre de 2021

Fecha de publicación: marzo de 2022

Pese a la alarma social que suscita el comportamiento delictivo en menores, los datos reflejan la baja prevalencia de estos hechos. No obstante, es y ha sido de suma importancia para la intervención social el estudio de modelos explicativos de dicho comportamiento, tanto por la elaboración de estrategias preventivas como por la intervención psicoeducativa con estos. El modelo de triple riesgo delictivo pretende explicar la etiología de estos comportamientos antisociales y delictivos, precisamente, para conseguir dichos fines y partiendo de diferentes modelos y teorías integradoras. Este trabajo pretende explicar este modelo en la práctica profesional con estos menores, y concluye con la aparición de las tres fuentes de riesgo: riesgos individuales, carencias prosociales y oportunidades delictivas en estos. El modelo de triple riesgo delictivo tiene en cuenta la acumulación de riesgos, pudiendo identificar la estimación de riesgo delictivo futuro.

\section{Palabras clave}

delincuencias menores; riesgo delictivo; factores de riesgo; menor en riesgo social 


\title{
Study of risk factors in juvenile offenders for the design of social intervention
}

\begin{abstract}
Despite the social alarm caused by criminal behaviour in minors, the data reflects the low prevalence of such behaviour. However, it is and has been of great importance for social intervention to study explanatory models of such behaviour, to develop both preventative strategies and psychoeducational interventions. The Triple Risk Crime Model aims to explain the aetiology of these antisocial and criminal behaviours, precisely in order to achieve this goal and based on different integrative models and theories. This study aims to explain this model in professional practice with these minors, concluding with the emergence of the three sources of risk: individual risks, prosocial deficiencies and criminal opportunities. The Triple Crime Risk model takes into account the accumulation of risks and so is able to identify the estimation of future risk of crime.
\end{abstract}

\section{Keywords}

minor crime, crime risk, risk factors. minor in social risk 


\section{Introducción}

Desde hace décadas, la intervención social quiere dar respuesta al problema social de la delincuencia en menores, ya que se considera circunscrita a una situación de desprotección, buscando además explicaciones para su comprensión.

En general, se ha estudiado, y se sigue estudiando con detalle, la delincuencia juvenil y, especialmente, el cambio de tipología delictiva que desarrollan. Aunque son muchos los que pretenden representar la delincuencia $y$, en concreto, la delincuencia juvenil como una alarma social, los datos no demuestran tal inseguridad objetiva, pero no deben minimizarse, ya que sí es un fenómeno complejo y genera una gran preocupación social, puesta de manifiesto en el barómetro del CIS (2019) que concluye que la inseguridad ciudadana es uno de los problemas principales de la sociedad, puesto que la sociedad española considera que puede afectarles directamente.

En cuanto a la intervención con menores delictivos, los últimos datos (2020) aportados por el Instituto Nacional de Estadística, a partir del Registro Central de Sentencias de Responsabilidad Penal de los menores, indican que 11.238 menores se encuentran bajo una medida judicial por la comisión de un hecho delictivo, lo que corresponde aproximadamente al 0,75\% de población española de edades comprendidas entre 14 y 17 años.

El propósito para comprender y explicar la delincuencia en menores, con el fin de adoptar medidas preventivas y de intervención, ha dado lugar a distintas líneas de investigación, con el objeto de considerar la relación de factores que influyen en dicho comportamiento delictivo.

La revisión realizada por Leblanc y Robert (2012) considera que un enfoque innovador en la prevención delictiva es aquel basado en la intervención en el ambiente, como son el entorno familiar y escolar y el barrio donde conviven (McCord et al., 2001), aunque recogen también la propuesta de Welsh y Farrigton (2007) en relación con la intervención en múltiples niveles, incluyendo la propuesta de McCord et al. (2001) referente a la intervención individual.

La responsabilidad penal del menor está regulada por la Ley Orgánica 5/2000, en la que se contemplan las actua- ciones del tipo sancionador y educativo que promueve el objeto de esta regulación, pero generalmente corresponde a las comunidades autónomas y, en concreto, a las competenciales de los servicios sociales la disposición de recursos para el cumplimiento de las medidas judiciales impuestas por los juzgados de menores. Aquellos comportamientos que se encuentran incluidos en nuestra legislación penal serán los que se consideren, en el caso de los menores, como delictivos, frente a otros comportamientos, como los desviados, al infringir normas sociales o de riesgo que puedan ser, en un futuro, problemáticos, como el consumo de drogas.

La premisa fundamental de este trabajo es que los menores con comportamientos delictivos se encuentran en situación de riesgo por la influencia de diferentes factores de riesgo. Se considera que la influencia entre los riesgos individuales, la falta de apoyo prosocial y la exposición a las oportunidades delictivas favorecen el riesgo global del comportamiento delictivo (Bobbio et al., 2021).

\section{Modelo de triple riesgo delictivo}

Diferentes estudios han conseguido identificar los factores de riesgo y protección que presentan mayor correlación con la conducta antisocial o delictiva en menores, pero especialmente la pretensión es y ha sido construir conglomerados configurados según las variables analizadas, que pudieran dar respuesta a la interacción de los diferentes factores de riesgo (Battin-Pearson et al., 1998; Vázquez, 2003; Graña y Biezma, 2010; Mampaso et al., 2014).

Redondo (2008) publicó la versión original del modelo del triple riesgo delictivo (MTRD), modelo explicativo sobre la delincuencia con el que pretendía establecer una estructura metateórica global, que incorporara distintas descripciones sobre la etiología de la delincuencia, tanto de los factores de riesgo para el delito como de otros modelos integradores de esta. El modelo niega la existencia de una única causa que origine el delito, considerando que son múltiples las causas que explican la delincuencia.

Redondo (2015) considera que, para que los individuos manifiesten inhibición sobre ciertos comportamientos delictivos, se precisan tres condiciones complementarias: 
- Disposiciones y capacidades personales favorables, como la inteligencia, las habilidades de comunicación, el autocontrol, etc., de las que está dotada, habitualmente, la mayoría de la población. Lo contrario serían los riesgos personales o características individuales, tanto congénitas como adquiridas, que complican los procesos comunes de desarrollo y socialización. Incluye las variables biológicas, las características individuales de las personas relacionadas con el pensamiento, el comportamiento y las situaciones traumáticas.

- Contar con apoyo prosocial suficiente y continuado de su familia, y de otros estamentos sociales, fundamentalmente en su infancia y juventud, pero también durante etapas posteriores de su vida. La identificación de tal factor de riesgo es la carencia de apoyos prosociales que entorpecen o frenan su socialización. Se considera que los individuos que experimenten mayores carencias en apoyo prosocial presentarán mayor vulnerabilidad en el momento de participar en actos delictivos. La confluencia de diversas carencias prosociales impulsará o favorecerá la conducta antisocial.

- Estar suficientemente protegido frente a numerosas e intensas oportunidades delictivas. El ambiente presenta oportunidades delictivas, situaciones o estímulos, tanto físicos como sociales, que facilitan las conductas infractoras. El incremento de la exposición de un individuo a objetivos y víctimas vulnerables aumentará la probabilidad de que cometan delitos. La suma de oportunidades contextuales para la comisión de actividades delictivas impulsará la aparición de otras oportunidades delictivas relacionadas.

El modelo de triple riesgo delictivo establece que la probabilidad individual de cometer un delito depende de la combinación e interacción, en un mismo sujeto, entre los riesgos personales, las carencias en apoyo prosocial y la exposición a oportunidades delictivas. Es fundamental considerar la combinación entre riesgos de distintas naturalezas, la convergencia de riesgos interfuentes y la potenciación recíproca de los riesgos interfuentes que aumentan la probabilidad individual de una conducta delictiva.

Otro concepto interesante en este modelo es el de la motivación delictiva, definido como el nivel de disposición individual para la comisión de conductas delictivas, incluyendo las creencias, los afectos o las conductas de aproximación a objetivos delictivos que manifieste el sujeto con anterioridad a la comisión de la infracción.
El modelo del triple riesgo delictivo (MTRD) de Redondo (2008) realiza un estudio profundo sobre la relación de los factores de riesgo agrupados en la explicación del origen, el mantenimiento y la inhibición de la conducta delictiva. El objetivo final es elaborar una explicación del comportamiento delictivo sobre la base de las tres fuentes de riesgo identificadas por Redondo (2008) en un determinado periodo de tiempo, considerando que este riesgo dependerá de las tres fuentes explicadas anteriormente. Análogamente a otros modelos explicativos, distingue entre factores estáticos y dinámicos, siendo los primeros difíciles de cambiar; a diferencia de los dinámicos, esta consideración es utilizada en los planes de intervención para provocar el cambio de la conducta antisocial a prosocial. El modelo, presentado por el profesor Santiago Redondo, se posiciona en el trabajo, no solo con los factores de riesgo, sino también con los de protección; por este motivo, en su modelo teórico se considera cada variable como un continuo, sin ocuparse de los polos opuestos, al estimar la graduación de cada una de las variables como un elemento clave, no solo en la prevención o el control del delito, sino, como podemos suponer, en lo referente a la valoración del riesgo delictivo.

El estudio de los factores de riesgo y protección, así como los modelos explicativos teniendo en cuenta estos factores, ha sido y es de gran interés en el estudio de la delincuencia. Diferentes autores han realizado distintas clasificaciones de los factores de riesgo; según Hawkins et al. (1992), se pueden distinguir entre los genéticos, los psicológicos y socioculturales. Peterson et al. (1992) distribuyen los factores de riesgo de la comunidad, incluyendo la privación económica y social, el bajo apego en la crianza y la desorganización comunitaria, los cambios o transiciones y la movilidad y la disponibilidad de las drogas. En factores de riesgo familiares se incluyen antecedentes familiares de drogodependencia, problemas de control por parte de la familia, el uso de drogas por vía parenteral y las actitudes positivas hacia su uso. Entre los factores de riesgo escolar podemos destacar una temprana conducta antisocial, el fracaso académico y un bajo compromiso con la escuela. Si atendemos a los factores de riesgo individual y con respecto a sus iguales, estos comprenden la alienación y la rebeldía, la conducta antisocial en la adolescencia temprana, los amigos que consumen drogas, las actitudes favorables hacia el consumo de drogas y los primeros consumos tempranos de drogas. 
Moncada (1997) diferencia entre factores de riesgo ambientales, como la deprivación social, la desorganización comunitaria, la disponibilidad y accesibilidad de la sustancia, la percepción social de riesgo, la movilidad de la población y las normas y leyes de la comunidad, así como los factores de riesgo del individuo y sus relaciones con el entorno, como una historia familiar de alcoholismo, las pautas educativas, las actitudes y modelos de conducta por parte de los padres, los conflictos familiares, los valores de agresividad, la búsqueda de sensaciones, los problemas de conducta, las actitudes favorables hacia las drogas, el fracaso escolar y el grupo de iguales consumidores. Muñoz-Rivas et al. (2002) realizan una revisión exhaustiva de los factores de riesgo y protección que agrupan en ambientales/contextuales, individuales (genéticos, biológico-evolutivos y psicológicos) y factores de socialización, así como la investigación de Lösel y Farrigton (2012) sobre los factores de protección, y la clasificación realizada por Mampaso et al. (2014).

En la actualidad, los factores de protección adquieren una gran relevancia, e incluso algunos instrumentos de riesgo actuales utilizan estos para la evaluación y gestión del riesgo en menores, cuyo objetivo es dotar de destrezas y estrategias de afrontamiento prosociales.

Este trabajo pretende analizar los factores de riesgo propuestos por el modelo de triple riesgo delictivo, evaluando a menores de edad que en el momento del estudio se encontraban cumpliendo una medida judicial en un recurso acreditado.

\section{Metodología}

\subsection{Participantes}

La población objeto de estudio son menores de edad que han cometido algún delito. Los criterios de inclusión para la participación del estudio ha sido ser menores de edad. Se seleccionaron para formar parte de la muestra menores de edad que acudían a un recurso específico para el cumplimiento de medidas judiciales de medio abierto en Salamanca. La muestra final estaba formada por 44 sujetos, cuya edad se encontraba entre 14 y 18 años ( $M=16,55$; DT $=1,49$ ). En su totalidad eran de nacionalidad española y principalmente hombres, siendo 8 mujeres y 36 hombres; es decir, un $19 \%$ corresponde a mujeres.

\subsection{Variables e Instrumentos}

Los datos fueron recogidos mediante el inventario de riesgos individuales y sociales revisado (IRIS-R) y diseñado por Redondo y Martínez-Catena (2014), según el modelo de triple riesgo delictivo (Redondo, 2008). Mediante las subescalas de este instrumento se evalúan las diferentes fuentes de riesgo propuestas por el autor en su modelo teórico a partir de una serie de indicadores. La subescala de riesgos personales incluye 11 indicadores; la de carencias prosociales 22 y 2 la de oportunidades delictivas. Además, se incluye la evaluación de las conductas infractoras y delictivas compuestas por 16 indicadores. En cada indicador, los participantes han de responder en una escala Likert comprendida entre 0 y 5 , según la percepción de cada uno de ellos, para cada ítem.

\subsection{Procedimiento}

Todos los casos fueron evaluados directamente por profesionales pertenecientes al centro de cumplimiento y se analizaron estadísticamente con anonimato mantenido.

\subsection{Análisis estadísticos}

Se han analizado las respuestas de los dos instrumentos, utilizando un nivel de significación $p \leq 0,05$ con los estadísticos siguientes:

- Análisis de descriptivos de cada uno de los ítems. Las puntuaciones de los resultados de cada uno de los ítems nos permiten obtener la media y desviación típica del grupo analizado.

- Análisis factorial como método de extracción de factores, Varimax como método de rotación y el criterio de Kaiser para la selección de factores, lo que nos permite analizar la aplicación del modelo de triple riesgo delictivo.

\section{Resultados}

Con la finalidad de conocer las variables más significativas en nuestros participantes según las tres fuentes de riesgo del modelo de Redondo (2008), se distingue entre rasgos biológicos, rasgos personales, carencias prosociales, oportunidades delictivas y comportamientos delictivos y antisociales. Como se puede observar en la Tabla 1, se 
ha encontrado prevalencia de bajo peso al nacer en los participantes analizados, situándose dichas variables en la fuente de riesgo personal. Los antecedentes familiares de adicciones tienen menor presencia.

\begin{tabular}{|l|l|}
\hline \multicolumn{2}{|c|}{ Riesgos biológicos } \\
\hline Bajo peso al nacer & $59,1 \%$ \\
\hline Problemas en el embarazo o parto & $15,9 \%$ \\
\hline Lesión grave en la cabeza & $6,8 \%$ \\
\hline Antecedentes de alcoholismo en la familia & $25 \%$ \\
\hline $\begin{array}{l}\text { Antecedentes de consumo de drogas en } \\
\text { la familia }\end{array}$ & $29,5 \%$ \\
\hline Fuente: elaboración propia & \\
\hline
\end{tabular}

Tabla 1. Riesgos biológicos

En nuestra investigación, como muestra la Tabla 2, los factores de riesgo personales de mayor presencia son las características de personalidad: la búsqueda de sensaciones y experiencias arriesgadas, además de la baja tolerancia a la frustración. Incluso puede observarse una tendencia a la impulsividad, la intranquilidad y el egocentrismo.

\begin{tabular}{|c|c|c|c|}
\hline Riesgos individuales & M & DT & $\begin{array}{l}\text { Mín.- } \\
\text { Máx. }\end{array}$ \\
\hline Riesgos biológicos & 1,18 & 1,18 & $0-4$ \\
\hline Impulsividad & 2,89 & 1,46 & $0-5$ \\
\hline Dificultad para estar tranquilo & 2,93 & 1,69 & $0-5$ \\
\hline $\begin{array}{l}\text { Atracción por experiencias estimulantes y } \\
\text { arriesgadas }\end{array}$ & 3,90 & 1,69 & $0-5$ \\
\hline Pensar en sí mismo & 2,89 & 1,70 & $0-5$ \\
\hline Frustración por no conseguir el objetivo & 3,11 & 1,70 & $0-5$ \\
\hline Falta de control ante el enfado & 2,50 & 1,62 & $0-5$ \\
\hline Justificación de delitos & 2,36 & 1,83 & $0-5$ \\
\hline Justificación de la violencia & 1,68 & 1,68 & $0-5$ \\
\hline Drogodependencia & 1,57 & 2,03 & $0-5$ \\
\hline Maltrato infantil y/0 en la adolescencia & 0,89 & 1,70 & $0-5$ \\
\hline
\end{tabular}

Tabla 2. Descriptivos en los indicadores de riesgos personales
En relación con las carencias prosociales, como se observa en la Tabla 3, los menores analizados han iniciado su carrera delictiva de manera precoz, ya que destaca la primera detención a los 14 años, y conviven en un barrio en el que hay más problemas sociales que en otros de la ciudad; en cuanto a la red social, el grupo de iguales está relacionado con la delincuencia. Respecto a la familia, destaca una tendencia a un entorno conflictivo con frecuentes discusiones y peleas. En estos menores sobresale su experiencia en entornos delictivos, que ellos mismos perciben como una influencia negativa.

\begin{tabular}{|l|l|l|l|}
\hline \multicolumn{2}{|c}{ Carencias prosociales } & \multicolumn{1}{c}{ DT } & \multicolumn{1}{c|}{ Mán. } \\
\hline Maternidad temprana & 0,39 & 0,72 & $0-2$ \\
\hline Número de hermanos & 1,50 & 1,53 & $0-5$ \\
\hline Grado de conflictividad familiar & 1,93 & 1,53 & $0-5$ \\
\hline Alcoholismo paterno & 0,80 & 1,63 & $0-5$ \\
\hline Alcoholismo materno & 0,45 & 1,28 & $0-5$ \\
\hline Delincuencia paterna & 0,32 &, 98 & $0-5$ \\
\hline Delincuencia materna & 0,09 &, 47 & $0-3$ \\
\hline Grado de discusiones y peleas & 1,50 & 1,53 & $0-5$ \\
\hline Violencia de género &, 59 & 1,41 & $0-5$ \\
\hline Delincuencia en el grupo de iguales & 2,30 & 1,93 & $0-5$ \\
\hline Primera detención & 2,48 & 1,32 & $0-5$ \\
\hline Frecuencia de detenciones &, 34 &, 91 & $0-5$ \\
\hline Primer ingreso en un centro de menores & 1,20 & 1,56 & $0-4$ \\
\hline Reincidencia de ingreso en el centro de menores &, 55 & 1,13 & $0-5$ \\
\hline Carencias en el barrio & 1,05 & 1,43 & $0-5$ \\
\hline Problemas en el barrio & 1,98 & 1,77 & $0-5$ \\
\hline Barrio de inmigrantes & 1,34 & 1,53 & $0-5$ \\
\hline Visualización de delitos graves & 1,5 & 1,67 & $0-5$ \\
\hline Influencia a la exposición de delitos graves & 1,48 & 1,95 & $0-5$ \\
\hline Relaciones de pareja tensas & 1,2 & 1,59 & $0-5$ \\
\hline Relaciones personales tensas & & & \\
\hline Pareja delincuente & 1,47 & $0-5$ \\
\hline Fuente: elaboración propia & & $0-5$ \\
\hline
\end{tabular}

Tabla 3. Descriptivos en los indicadores de riesgos personales 
Los resultados, como muestra la Tabla 4, indican que, en cuanto a las oportunidades de exposición delictiva, los participantes del estudio han estado en contacto con claras oportunidades para cometer delitos.

\begin{tabular}{|l|l|l|l|}
\hline \multicolumn{1}{|c|}{ Oportunidades delictivas } & \multicolumn{1}{|c|}{$\boldsymbol{M}$} & \multicolumn{1}{|c|}{ DT } & \multicolumn{1}{c|}{$\begin{array}{c}\text { Mín.- } \\
\text { Máx. }\end{array}$} \\
\hline Exposición a oportunidades delictivas & 2,61 & 1,75 & $0-5$ \\
\hline Cantidad de oportunidades delictivas & 1,18 & 1,46 & $0-5$ \\
\hline Fuente: elaboración propia & \multicolumn{3}{|l}{} \\
\hline
\end{tabular}

Tabla 4. Resultados de oportunidades de exposición delictiva

Observando la Tabla 5 y analizando las conductas infractoras y delictivas realizadas por los menores, destacan los comportamientos antisociales o desviados frente a los comportamientos delictivos como el inicio precoz del consumo de drogas legales e ilegales y el mantenimiento de dichos consumos. Aunque merece señalar la tendencia de estos, en el inicio de la carrera delictiva, a comportamientos delictivos menos graves, como son los hurtos, las agresiones y las lesiones. En este contexto, se puede considerar su prevalencia en la realización de comportamientos violentos sobre los compañeros del colegio en edades tempranas.

\begin{tabular}{|l|l|l|l|}
\hline \multicolumn{1}{|c|}{ Conductas antisociales y delictivas } & \multicolumn{1}{|c|}{ M } & \multicolumn{1}{|c|}{ DT } & $\begin{array}{l}\text { Mín.- } \\
\text { Máx. }\end{array}$ \\
\hline Pegar a compañeros & 1,25 & 1,65 & $0-5$ \\
\hline Hurtos a iguales & 1,7 & 1,6 & $0-5$ \\
\hline Dañar a animales &, 39 &, 84 & $0-3$ \\
\hline Pellas en el colegio & 2 & 1,81 & $0-5$ \\
\hline Consumo de alcohol y drogas en la adolescencia & 2,89 & 1,8 & $0-5$ \\
\hline Edad de la primera borrachera & 2,8 & 1,47 & $0-5$ \\
\hline Edad de consumo de drogas & 2,52 & 1,63 & $0-5$ \\
\hline Frecuencia delictiva &, 64 & 1,05 & $0-4$ \\
\hline Frecuencia de condenas &, 61 & 1,01 & $0-3$ \\
\hline Hurtos & 1,23 & 1,75 & $0-5$ \\
\hline Robos con fuerza &, 61 & 1,35 & $0-5$ \\
\hline Robos con intimidación &, 59 & 1,36 & $0-5$ \\
\hline
\end{tabular}

\begin{tabular}{|l|l|l|l|}
\hline Agresiones y lesiones & 1,05 & 1,55 & $0-5$ \\
\hline Venta de drogas &, 57 & 1,28 & $0-5$ \\
\hline Homicidios &, 00 &, 00 & $0-0$ \\
\hline Comisión de otros delitos &, 50 &, 97 & $0-5$ \\
\hline Fuente: elaboración propia & \multicolumn{3}{|l}{} \\
\hline
\end{tabular}

Tabla 5. Descriptivos de los indicadores de los comportamientos antisociales y delictivos

\section{Conclusiones}

Tras el estudio realizado, en nuestros participantes destacan los factores de riesgo personales relacionados con el bajo peso al nacer, la necesidad de sobreestimulación y experiencias de riesgo, baja tolerancia a la frustración y, en menor medida, la característica personal de egocentrismo e impulsividad. En cuanto a las carencias prosociales, destacan el grupo de iguales relacionados con la delincuencia y la primera detección a partir de los 14 años, además de una tendencia a oportunidades delictivas que explicarían, en su conjunto, el inicio y mantenimiento de la carrera delictiva, en nuestro caso de menor gravedad. En relación con las conductas delictivas más frecuentes, destaca el inicio precoz de consumo de alcohol y drogas.

En los resultados más relevantes obtenidos respecto a la fuente de riesgo personal, destacan aquellas características que han demostrado una mayor correlación en los estudios sobre el comportamiento delictivo. La investigación realizada por López-Romero et al. (2019) distingue perfiles en los menores infractores según los riesgos presentes, identificando un perfil denominado impulsivo o subcontrolado de alto riesgo. Presentan, en este caso, una personalidad característica y un patrón de riesgo actitudinal caracterizado por altos niveles de riesgo de suicidio, impulsividad, egocentrismo, atribución externa de responsabilidad, actitudes positivas a la violencia y deficientes habilidades sociales.

Farrington (1992), en su teoría integradora, considera como factor influyente en el comportamiento delictivo la falta de empatía que facilita la personalidad egocentrista; en nuestro estudio, los menores analizados presentan un patrón similar. 
En referencia a la fuente de riesgo de carencias prosociales, en nuestra investigación destaca la prevalencia de la red social delictiva de los participantes. Investigaciones relacionadas con el grupo de iguales han demostrado la influencia de este en la adquisición de actitudes y comportamientos antisociales y delictivos (Moreira y Mirón, 2013; Andrews y Bonta, 2010; Lonardo et al., 2009; Granic y Patterson, 2006). El estudio realizado por Cutrin et al. (2015) demuestra que los vínculos establecidos con el grupo de iguales son predictores en el comportamiento desviado y antisocial, de la misma forma que ha tenido su influencia en este asunto el estudio realizado por Dishion et al. (2010).

El trabajo realizado por Margolin y Baucom (2014) obtuvo resultados que mostraban el impacto negativo de la exposición de la violencia en tres ámbitos diferentes (la violencia filioparental, la agresión entre los cónyuges y la violencia comunitaria) y en tres años, en nuestro estudio, los menores reconocen estar expuestos a situaciones delictivas manifestadas en diferentes problemáticas sociales, lo que influye en su propio comportamiento delictivo. Según De Antoni y Koller (2002), este comportamiento construido en el entorno familiar explica los patrones establecidos en otros entornos sociales fuera del escenario familiar.

Bonta y Andrews (2016) consideran que el comportamiento delictivo no está predeterminado. El contexto controla la manifestación del resto de las variables, como los rasgos de personalidad, dependiendo de estas en la interacción con el ambiente.

En relación con las conductas identificadas como desviadas por el autor del MTRD, se encuentra el consumo de drogas precoz y el inicio temprano de la carrera delictiva. En ambos casos, la investigación predice el denominado «efecto escalada» más habitual utilizado en el caso de la prevención a las adicciones, relacionado, entre otras variables, con la legitimación cultural de los consumos y la baja percepción de riesgo (Calafat et al., 2002), y el caso del comportamiento delictivo relacionado con la curva de la edad del delito (Farrington, 1986; Piquero et al., 2007), en la que se observa una generalización de los comportamientos antisociales y delictivos en el transcurso de algunas edades. Vega y Zumárraga (2018), al analizar las variables asociadas a la conducta delictiva y adictiva en su estudio, observaron una correlación entre los sujetos que tuvieron conflictos previos con la ley y un mayor consumo de sustancias adictivas; dichos resultados coincidieron con investigaciones como la de Marshall et al. (1990) y fueron contrarios a la de Gallo (2015), por lo que se propone profundizar en este aspecto en futuras líneas de investigación.

A pesar de las ventajas obtenidas en nuestro estudio, en relación con los factores influyentes en la muestra, este no se encuentra exento de limitaciones. El número y las características de la muestra nos aportan un perfil de los menores atendidos en medio abierto en la provincia de Salamanca, siendo de interés ampliar la investigación con menores que se encuentren cumpliendo medidas judiciales privativas de libertad, incluso haciendo diferenciaciones entre las distintas medidas comunitarias.

Tal y como postula el modelo de triple riesgo delictivo (Redondo, 2008), la conducta delictiva no puede ser explicada por los factores de riesgo aislados (Gallo y WiIliams, 2005), de ahí que no solo sea importante tener en cuenta el contexto (Ludke y Dalbosco, 2010), sino también la interacción entre las diferentes variables de las que la academia ha demostrado su correlación, lo que se aplica a la pretensión del modelo de triple riesgo delictivo.

La identificación de factores de riesgo no solo facilita la explicación etiológica del comportamiento de estos menores, sino que, además, nos ayudará a diseñar programas de intervención orientados a estos factores identificados, en este caso, en relación con estrategias de autocontrol, habilidades sociales, estrategias para combatir la presión grupal o intervención preventiva en adicciones. 


\section{Referencias bibliográficas}

ANDREWS, D. A.; BONTA, J. (2010). «Rehabilitating criminal justice policy and practice». En: Psychology, Public Policy, and Law, vol. 16, núm. 1, págs. 39-55. DOI: https://doi.org/10.1037/a0018362

BATTIN-PEARSON, S.; TERENCE, P.; THORNBERRY, J.; HAWKINS, D.; KROHN, M. (1998). «Gang Membership, Delinquent Peers, and Delinquent Behavior». En: Juvenile Justice Bulletin, NCJ 171119, págs. 1-10. DOI: https://doi.org/10.1037/e304542003-001

BOBBIO, A.; ARBACH, K; REDONDO, S. (2021) «El Modelo Triple Riesgo Delictivo en la explicación de la conducta antisocial de adolescentes varones y mujeres». En: Revista Española de Investigación criminológica, vol. 6, núm. 19. DOl: https://doi.org/10.46381/reic.v19i1.479

BONTA, J.; ANDREWS, D. (2016). The psychology of criminal conduct. Routledge. DOI: https://doi. org/10.4324/9781315677187

CALAFAT, A.; JUAN, M.; BECOÑA, E.; FERNÁNDEZ, C.; GIL, E.; LLOPIS, J. (2002). «Organización y estrategias de la cultura pro-cannabis». En: Adicciones, vol. 12, núm. 2 Supl, págs. 231-273. http://dx.doi. org/10.20882/adicciones.683

CUTRÍN, O.; GÓMEZ-FRAGUELA, J. A.; LUENGO, M. A. (2015). «Peer-group mediation in the relationship between family and juvenile antisocial behavior». En: The European Journal of Psychology Applied to Legal Context, vol. 7, núm. 2, págs. 59-65. DOI: https://dx.doi.org/10.1016/j.ejpal.2014.11.005

DE ANTONI, C.; KOLLER, S. (2002). «Violencia doméstica y Comunidad». En M. Contini, S. Koller y M. Barros (eds.), Adolescencia y psicología: conceptos, prácticas críticas y reflexiones críticas, págs. 85-91. Consejo Federal de Psicología.

DISHION, T.; VERONNEAU, M.; MYERS, M. (2010). «Cascading peer dynamics underlying the progression from problem behavior to violence in early to late adolescence». En: Development and Psychopathology, vol. 22, núm. 3, págs. 603-619. DOI: https://doi.org/10.1017/S0954579410000313

FARRINGTON, D. (1992) La reeducación del delincuente juvenil. Los programas de éxito. Tirant lo Blanch.

GALLO, A.; WILLIAMS, L. (2005). «Adolescentes en conflicto Ley: una revisión de los factores de riesgo para la anticoncepción conducto de infracción». En: Psicología: teoría y práctica, vol. 7, núm. 1, págs. 81-95

GALLO, K. (2015). The relationship between age of onset of drug use, drug dependence, mental disorders, and offense type and severity, (Tesis doctoral, California State University) [en línea]. Disponible en: https://scholarworks.lib.csusb.edu/cgi/viewcontent.cgi?article=1141\&context=etd

GRAÑA, J.; RODRÍGUEZ, M. (2010). «Programa central de tratamiento educativo y terapéutico para menores infractores». Agencia de la Comunidad de Madrid para la Reeducación y Reinserción del Menor Infractor.

GRANIC, I.; PATTERSON, G. R. (2006). «Toward a comprehensive model of antisocial development: A dynamic systems approach». En: Psychological Review, vol. 113, núm. 1, págs. 101-131. DOI: https:// doi.org/10.1037/0033-295X.113.1.101

HAWKINS, J.; CATALANO, R.; MILLER, J. (1992). «Risk and protective factors for alcohol and other drug problems in adolescence and early adulthood: Implications for substance abuse prevention». En: Psychological Bulletin, vol. 112, núm. 1, págs. 64-105. DOI: https://doi.org/10.1037/0033-2909.112.1.64

INSTITUTO NACIONAL DE ESTADÍSTICA (2018). Estadística de condenados menores [en línea]. Disponible en: https://www.ine.es/dynt3/inebase/index.htm?padre=3977\&capsel=3977 
LEBLANC, L.; ROBERT, M. (2012). «La innovación psicosocial: planificar su implementación y difusión para prevenir la delincuencia juvenil». En: Universitas Psychologica, vol. 11, núm 4, págs. 1125-1134 [en línea]. Disponible en: http://www.scielo.org.co/scielo.php?script=sci_arttext\&pid=\$1657-92672012000400008\&lng=en\&tIng=es. DOI: https://doi.org/10.11144/Javeriana.upsy11-4.ippi

Ley Orgánica 5/2000, de 12 de enero, reguladora de la responsabilidad penal de los menores. Boletín Oficial del Estado, Madrid, de 13 de enero de 2000, núm. 11, págs. 1422-1441.

LONARDO, R. A.; GIORDANO, P.C.; LONGMORE, M. A.; MANNING, W.D. (2009). «Parents, friends, and romantic partners:Enmeshmentindeviantnetworksandadolescent delinquencyinvolvement». En:Journalof Youth Adolescence, vol.38, núm.3, págs.367-383.DOl:https://psycnet.apa.org/doi/10.1007/s10964-008-9333-4 https://doi.org/10.1007/s10964-008-9333-4

LÓPEZ-ROMERO, L.; MANEIRO, L; CUTRIN, O.; GÓMEZ-FRAGUELA, L.; VILLAR, P.; LUENGO, M.; SOBRAL, J.; ROMERO, E. (2019). «Identifying Risk Profiles for Antisocial Behavior in a Spanish Sample of Young Offenders». En: International Journal of Offender Therapy and Comparative Criminology, vol. 63, núm. 10, págs. 1896-1913. DOI: https://doi.org/10.1177/0306624X19842032

LÖSEL, F.; FARRINGTON, D. (2012). «Direct protective and buffering protective factors in the development of youth violence». En: American Journal of Preventive Medicine, vol. 43, núm. 2, págs. 8-23. DOI: https://doi.org/10.1016/j.amepre.2012.04.029

LUDKE, F.; DALBOSCO, D. (2010). «Delincuencia juvenil: Una revisión teórica». En: Acta Colombiana de Psicología, vol. 13, núm. 2, págs. 69-77.

MAMPASO, J.; PÉREZ, F.; CORBÍ, B.; GONZÁLEZ, P.; BERNABÉ, B. (2014). «Factores de riesgo y de protección en menores infractores». En: Análisis y prospectiva. Psychologia Latina, vol. 5, núm. 1, págs. 11-20.

MARGOLIN, G.; BAUCOM, B. R. (2014). «Adolescents' aggression to parents: Longitudinal links with parents' physical aggression». Journal of Adolescent Health, núm. 55, págs. 645-651. DOI: http:// dx.doi.org/10.1016/j.jadohealth.2014.05.008

MARSHALL, W.; LAWS, D.; BARBAREE, H. (1990). «Issues in sexual assault». En: W. Marshall, D. Laws y H. Barbaree (eds.), Handbook of sexual assault: Issues, theories, and treatment of the offender, págs. 3-8. Springer US. DOI: https://doi.org/10.1007/978-1-4899-0915-2

MCCORD, J.; WIDOM, C.; CROWELL, N. (2001). Juvenile crime, Juvenile Justice. Panel on juvenile crime: Prevention, treatment, and control. National Academy Sciences.

MONCADA, S. (1997). «Factores de riesgo y de protección en el consumo de drogas». En: P.N.D. (ed.), Prevención de las drogodependencias. Análisis y propuestas de actuación, págs. 108-129). Delegación del Gobierno para el Plan Nacional sobre Drogas.

MOREIRA, V. MIRÓN, L. (2013). «El papel de la identidad de género en la conducta antisocial de los adolescentes». En: Psicothema, vol. 25, núm. 4, págs. 507-513. DOI: https://doi.org/10.1037/t15755-000

MUÑOZ-RIVAS, M.; GRAÑA, J.; PEÑA, M.; ANDREU, J. (2002). «Influencia de la conducta antisocial en el consumo de drogas ilegales en población adolescente». En: Adicciones, vol. 14, núm. 3, págs. 313-320. DOI: https://doi.org/10.20882/adicciones.486

OSGOOD, D.; WILSON, J.; O'MALLEY, P.; BACHMAN, J.; JOHNSTON, L. (1996) «Routine activities and individual deviant behavior.» En: American Sociological Review, vol. 61, núm. 4, págs. 635-655. DOI: https://doi.org/10.2307/2096397 
PETERSON, P.; HAWKINS, J.; CATALANO, R. (1992). «Evaluating comprehensive community drug risk reduction interventions. Design challenges and recommendations». En: Evaluation Review, vol. 16, núm. 6, págs. 579-602. DOI: https://doi.org/10.1177/0193841X9201600601

PIQUERO, A.; FARRINGTON, D.; BLUMSTEIN, A. (2007). Key issues in criminal career research: New analyses of the Cambridge Study in Delinquent Development. Cambridge University Press. DOI: https://doi.org/10.1017/CB09780511499494

REDONDO, S. (2008). «Individuos, sociedades y oportunidades en la explicación y prevención del delito: Modelo del Triple Riesgo Delictivo (TRD)». En: Revista Española de Investigación Criminológica, núm. 6, págs.1-53 [en línea]. Disponible en: https://reic.criminologia.net/index.php/journal/article/view/34 DOI: https://doi.org/10.46381/reic.v6i0.34

REDONDO, S. (2015). El Origen de los Delitos. Valencia: Tirant Humanidades.

VÁZQUEZ, C. (2003). Delincuencia juvenil. Consideraciones penales y criminológicas. Colex Editorial.

VEGA, J.; ZUMÁRRAGA, F. (2019). «Variables Asociadas al Inicio y Consumo Actual de Sustancias en Adolescentes en Conflicto con la Ley». En: Anuario de Psicología Jurídica, vol. 29, núm. 1, págs. 21-29. DOI: https://doi.org/10.5093/apj2018a13

WELSH, B.; FARRINGTON, D. (2007). «Scientific support for early prevention of delinquency and later offending». En: Victims \& Offenders, vol. 2, núm. 2, págs. 125-140. DOI: https://doi. org/10.1080/15564880701263114

\section{Cita recomendada}

YURREBASO MACHO, Amaia; PICADO VALVERDE, Eva M.a; ORGAZ BAZ, Begoña (2022). «Estudio de los factores de riesgo en menores infractores para el diseño de intervención social». IDP. Revista de Internet, Derecho y Política, núm. 35. UOC [Fecha de consulta: dd/mm/aa]

http://dx.doi.org/10.7238/idp.v0i35.387151

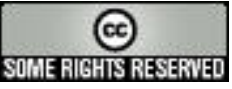

Los textos publicados en esta revista están -si no se indica lo contrario- bajo una licencia Reconocimiento-Sin obras derivadas 3.0 España de Creative Commons. Puede copiarlos, distribuirlos y comunicarlos públicamente siempre que cite su autor y la revista y la institución que los publica (IDP. Revista de Internet, Derecho y Política; UOC); no haga con ellos obras derivadas. La licencia completa se puede consultar en: http://creativecommons.org/ licenses/by-nd/3.0/es/deed.es. 


\section{Sobre los autores}

\section{Amaia Yurrebaso Macho}

Universidad de Salamanca

amaiay@usal.es

Profesora del departamento de Psicología Social y Antropología, Universidad de Salamanca. Doctora en Psicología por la Universidad de Salamanca, ha compatibilizado su actividad profesional como formadora de empresa y consultora freelance en el ámbito de la gestión y desarrollo de personas. Sus líneas de investigación son emprendizaje y colectivos desfavorecidos, cultura organizativa y grupos de trabajo, y violencia y riesgos psicosociales en entornos organizativos.

\section{Eva M. ${ }^{a}$ Picado Valverde}

Universidad de Salamanca

evapoicado@usal.es

Profesora del Departamento de Derecho del Trabajo, Trabajo Social y Servicios Sociales de la Universidad de Salamanca. Doctora en Psicología. Máster en Adicciones, clínico, forense y mediación penal. Tiene experiencia como psicóloga en la intervención con diferentes colectivos: menores en situación de desprotección, menores infractores, drogodependencia y reclusos. Técnico superior en igualdad de género y agente de igualdad de oportunidades vinculada a diferentes organizaciones del tercer sector en el ámbito nacional, regional y local. Premio nacional Victoria Kent (2016).

\section{Begoña Orgaz Baz}

Universidad de Salamanca

borgaz@usal.es

Profesora del Área de Metodología de las Ciencias del Comportamiento. Departamento de Psicología Básica, Psicobiología y Metodología de las Ciencias del Comportamiento. Facultad de Psicología de la Universidad de Salamanca. Doctora en Psicología, miembro del grupo de investigación sobre sexualidad y relaciones interpersonales. Participa en investigaciones sobre discapacidad. Es miembro de diferentes grupos de investigación dentro del campo de la discapacidad (inclusión educativa y ambiente, participación y calidad de vida, Grupo de INICO). Colabora en los proyectos de investigación de estos grupos, y centra su contribución en los aspectos metodológicos de diseño de la investigación, preparación y análisis de los datos y presentación de resultados.

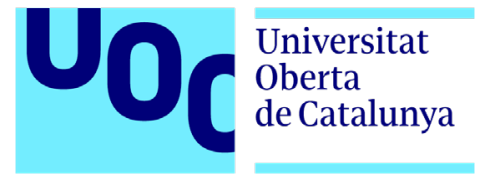

